\title{
El Tracto Cortico Espinal: Perspectiva Histórica
}

\author{
The Corticospinal Tract: Historical Perspective
}

\author{
Jorge Eduardo Duque Parra ${ }^{1,2,3}$; Julián Mendoza Zuluaga ${ }^{1,3}$ \& John Barco Ríos ${ }^{1,3}$
}

DUQUE, P. J. E.; MENDOZA, Z. J. \& BARCO, R. J. El tracto cortico espinal: perspectiva histórica. Int. J. Morphol., 38(6):1614$1617,2020$.

RESUMEN: La neuroanatomía y la neurofisiología han permitido en gran parte entender de forma más integrada las estructuras que conforman el sistema nervioso y los mecanismos asociados con la transmisión de los potenciales de acción, relacionados con la vía corticoespinal en la ejecución de movimientos voluntarios. Se realizó una revisión histórica sobre la vía corticoespinal, desde el punto de vista neuroanatómico y neurofisiológico mediante una revisión de literatura en distintas bases de datos y libros de texto dedicados a estas vías nerviosas. La información obtenida se ordenó cronológicamente, seleccionando los datos más relevantes que desde el punto de vista neuroanatómico y neurofisiológico han permitido comprender su mecanismo funcional. Actualmente se tiene un conocimiento muy depurado de los distintos elementos que componen la vía corticoespinal, lo que permitirá su aplicación en el campo de la salud y resolver múltiples problemas de la función motora.

PALABRAS CLAVE: Historia; Neuroanatomía; Tracto corticoespinal; Neurofisiología; Motoneuronas

\section{INTRODUCCIÓN}

Varias disciplinas contribuyen al conocimiento integral del sistema nervioso y, por tanto, al conocimiento de los seres vivos, que desde épocas remotas han aportado con sus hallazgos a dicho entendimiento, asociado invariablemente con la alteración neurológica (Duque-Parra, 2002). Uno de estos grandes aportes ha sido el conocimiento detallado de la vía motora, a través de la cual se transmite la información nerviosa para la ejecución y control de las acciones voluntarias musculares (Zinger et al., 2013). En los mamíferos, incluido el ser humano, dicha vía involucra los circuitos nerviosos necesarios para que los potenciales de acción, sean transmitidos desde la corteza cerebral hasta la médula espinal y, finalmente, desde ésta hacia los músculos esqueléticos (BravoAmbrosio et al., 2012) a través de los axones de las motoneuronas alfa.

Historia. Fue Hipócrates (460-380 a.n.e) el primero en referirse a la naturaleza decusada de la vía motora, aun sin tener un conocimiento claro de ella, hecho que se evidencia en su siguiente escrito: "Si la herida era situada en el lado izquierdo (de la cabeza) los ataques de convulsiones se daban en la mitad derecha del cuerpo humano" (Finger, 1994). Hipócrates notó que las lesiones de un lado de la cabeza a menudo produ- cían parálisis del lado contralateral del cuerpo. Posteriormente, Areteo de Capadocia (120-200 a.n.e), refiriéndose a lo manifestado por Hipócrates, dijo que esta característica debía obedecer a un cruce en alguna parte de la vía. Casi dos mil años después, Thomas Willis (1621-1675), en su libro Cerebri anatome, describió en 1664 las pirámides de la médula oblonga (Al Masri, 2011; Arráez-Aybar et al., 2015). Pero aún más que esta descripción, fue el hallazgo que hicieron Francois Pourfour du Petit (1664-1741) y Doménico Mistichelli (16751715) (Finger), quienes lograron identificar, en la parte inferior de la médula oblonga, el sitio donde ocurre la decusación de los tractos motores de la vía corticoespinal, vinculada con la motricidad voluntaria (Davidoff, 1990; Bennett \& Hacker, 2008), tanto en humanos como en otros primates. Mistichelli propuso, además, que el cruce nervioso que se da en las pirámides (Fig. 1) permitía explicar la causa de la hemiplejia, seguida de daño del lado opuesto de la cabeza; sin embargo, erróneamente creía que los nervios se originaban de la duramadre. Por otro lado, Pourfour realizó sus observaciones en soldados que presentaban parálisis, pero también experimentó con perros (Finger), a los que les provocó lesiones controladas con el fin de determinar qué región neuroanatómica se correlaciona con la función orgánica (Duque Parra et al.,

\footnotetext{
1. Universidad de Caldas. Facultad de Ciencias para la salud. Departamento de Ciencias Básicas. Manizales. Colombia.

2. Universidad de Manizales. Facultad de Ciencias para la salud. Departamento de Ciencias Básicas. Manizales. Colombia.

3. Universidad de Caldas. Departamento de Ciencias Básicas. Grupo Neurociencia de Caldas. Manizales. Colombia.
} 
2014). Tiempo después, se determinó que el tracto corticoespinal corresponde a un gran paquete de axones muy largos que se originan de neuronas piramidales, las cuales se caracterizan por presentar somas voluminosos con muchas ramificaciones dendríticas. Dichas neuronas se localizan particularmente en las capas V y III del córtex frontal motor primario -área citoarquitectónica 4- (Jacobs et al., 2018), y difieren en sus conexiones sinápticas y número de dendritas (Markram et al., 1998).

Dichas neuronas fueron descritas por Vladimir Alexandrovich Betz (1834-1894) en primates (Jacobs et al.), y tradicionalmente en la enseñanza de la neuroanatomía, se ha indicado que son aproximadamente 33300 (Afifi \& Bergman, 2006) o que contribuyen con 30000 axones a la formación del fascículo corticoespinal de cada lado (Kiernan \& Rajakumar, 2014), pero trabajos experimentales han demostrado que en realidad hay 125290 neuronas en promedio (Rivara et al., 2003). Los clínicos alemanes Gustav Theodor Fritsch (1838-1927) y Eduard Hitzig (1839-1907) descubrieron, en 1870, al estimular la corteza cerebral frontal en perros, mediante corriente eléctrica, que se daban discretos movimientos del cuerpo (Gross, 2007). Además, Fritsch había comprobado, durante la guerra pruso-danesa, que al limpiar con un paño zonas expuestas del cerebro de soldados heridos aparecían ocasionalmente contracciones en la parte opuesta del cuerpo. Estas observaciones contradecían la creencia general que se tenía por aquella época, a raíz de los trabajos experimentales realizados por el fisiólogo Marie Jean Pierre Fluorens (1794-1867) unos 40 años atrás. Pierre Fluorens había estimulado directamente el cerebro de palomas y otros animales y no observó ninguna respuesta cortical, por lo que concluyó que la corteza cerebral era inerte con relación a las funciones motoras o de cualquier otro tipo (Lazar, 2009). Pos-

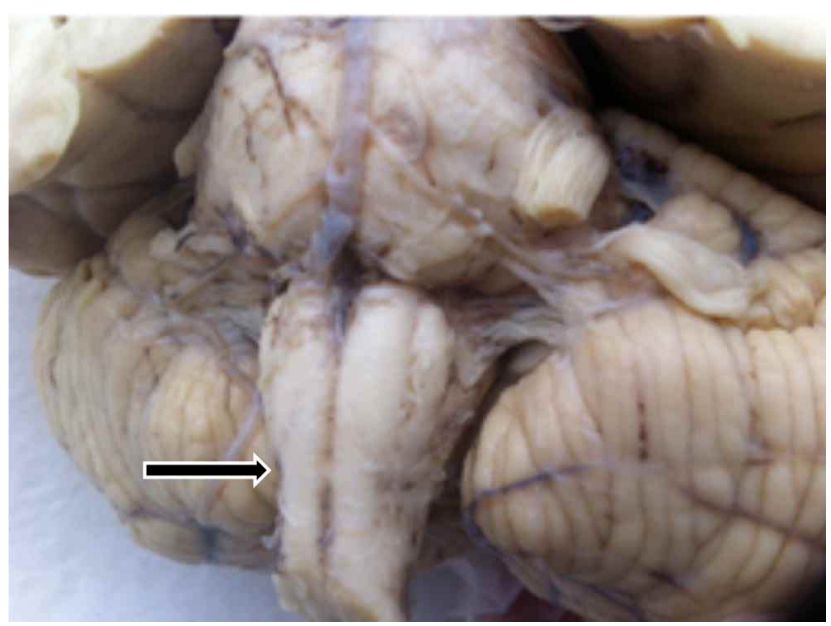

Fig. 1. Parte del tronco encefálico, donde se observan las pirámides en la médula oblonga y en el sector mediando la discontinuidad de la fisura mediana anterior -flecha negra-, evidenciándose allí la decusación piramidal. teriormente, en 1876, el neurólogo escocés David Ferrier (1843-1928) realizó extensos trabajos en simios, mostrando que existía una organización topográfica de los músculos del cuerpo en la corteza cerebral (Gross; Lazar). En los primeros años del siglo XX, los neurofisiólogos Charles Sherrington (1857-1952), Cecile Vogt (1875-1962) y Oscar Vogt (18701959), además de los neurocirujanos Otfrid Foerster (18731941) y Wilder Penfield (1891-1976), trabajando de manera independiente, realizaron importantes trabajos en perros, simios y humanos (Finger). Por ejemplo, Sherrington mostró que la destrucción de la región cortical del cerebro en el perro, rica en células piramidales, se asociaba con degeneración de su tracto piramidal. Sin embrago, Constantin von Monakov (1853-1930) argumentó que una pequeña porción del tracto corticoespinal provenía de los lóbulos parietal y frontal, dos áreas que Betz nunca encontró (Finger). Recientemente, la vía corticoespinal en humanos ha sido explorada mediante otra técnica no invasiva de difusión por tensión tractográfica (Holodny, 2005; Szmuda et al., 2020).

Visión neuroanatómica. Los tractos motores del sistema nervioso central humano pueden ser clasificados en corticoespinales y no corticoespinales (Davidoff). Se han descrito 3 tractos corticoespinales independientes: dos corticoespinales laterales, uno de los cuales es cruzado y otro no cruzado, y un tracto corticoespinal anterior, que es mayoritariamente ipsilateral (Davidoff.; Nyberg-Hansen \& Rinvik, 1963), aunque se han reportado fibras cruzadas que también van por el tracto corticoespinal anterior (Nathan et al., 1990). Así pues, el tracto corticoespinal lateral es mayoritariamente cruzado, mientras que el anterior es mayoritariamente ipsilateral (Al Masri). En un estudio realizado en cerebros humanos, se reportó la presencia de fibras corticoespinales laterales no cruzadas en el $10 \%$ de los casos, pero se desconoce su función, aunque estudios sobre evolución de este tracto sugieren que es exclusivo de mamíferos y que su desarrollo se asocia con adquisición de habilidades motoras diestras (Lemon \& Griffiths, 2005). Las fibras pertenecientes al tracto corticoespinal, que proceden de la corteza motora primaria, poseen una organización homuncular motora, con distintas regiones corporales allí representadas (Hooks et al., 2018). Dichas fibras descienden por la corona radiada y pasan por la mitad anterior del brazo posterior de la capsula interna, emitiendo colaterales para muchas estructuras subcorticales (Nyberg-Hansen \& Rinvik). Estas fibras descienden formando la base del pedúnculo cerebral, que luego pasan por el puente en cercanía con los núcleos pontinos, de gran influencia contralateral para el movimiento de los miembros (Hooks et al.). En la parte inferior de la medula oblonga ocurre la decusación de las pirámides (Fig. 1), donde el 75-90 $\%$ de las fibras se cruzan a los cordones contralaterales formando el tracto corticoespinal lateral. Un pequeño porcentaje de tales fibras, entre el 10-25\%, permanecen ipsilaterales y 
forman el tracto corticoespinal anterior (Davidoff), que en la mayoría de las veces solo desciende hasta los segmentos torácicos superiores. El tracto corticoespinal anterior permite el control de la musculatura proximal del cuello, tronco y miembros superiores (Davidoff; Nyberg-Hansen \& Rinvik). La mitad derecha de la médula espinal es siempre más grande que la izquierda, debido a que la decusación se da en forma asimétrica. Esto significa que la cantidad de fibras que va del hemisferio cerebral izquierdo a la mitad derecha de la medula espinal es mayor, comparada con la cantidad de fibras que va del hemisferio cerebral derecho a la mitad izquierda de la medula espinal; sin embargo, esto no afecta la lateralidad. La vía corticoespinal es la vía motora más importante para la motricidad voluntaria en el ser humano y, en términos filogenéticos, es una vía joven, pues ha sido encontrada solamente en mamíferos; en roedores y marsupiales la vía corticoespinal proporciona la vía más directa sobre la cual la corteza cerebral controla el movimiento, influenciando en gran medida las interneuronas de las astas posteriores. Sin embargo, al ascender la escala filogenética a través de carnívoros y primates, el número de axones corticoespinales crece y las terminaciones corticoespinales se desplazan progresivamente hacia las interneuronas de la zona intermedia y de las astas anteriores, formando finalmente un número creciente de terminaciones sinápticas directamente en las propias motoneuronas. Con base a esta tendencia filogenética, se cree que los humanos tienen sinapsis corticomotoneuronales más directas que cualquier otra especie, lo que es consistente con las observaciones de que los humanos sufren una pérdida de motilidad más extensa por las lesiones del tracto corticoespinal que otros mamíferos (Schieber, 2007). Esta vía está particularmente desarrollada en primates y algunas otras especies, permitiendo movimientos precisos en los miembros (Eyre et al., 2001). El desarrollo y maduración de la vía corticoespinal ocurre durante la embriogénesis y la primera infancia, donde además se lleva a cabo el refinamiento de sus conexiones y la mielinización de las fibras. Estos eventos facultan la desaparición de los reflejos primigenios y la aparición de la motricidad fina, asociados con una disminución en la cantidad de fibras nerviosas que permanecen ipsilaterales (Weavil et al., 2016).

Visión neurofisiología. Los tractos corticoespinales están conformados por un número promedio de 1026630 fibras nerviosas (Rivara et al.), cuya mayoría se encuentran mielinizadas (Nyberg-Hansen \& Rinvik), como se ha evidenciado mediante resonancia magnética (Rothwell et al., 1987) incluso en ratas y macacos (Kraskov et al., 2019). La mielinización que presentan estas fibras motoras permite que los impulsos nerviosos se conduzcan a altas velocidades (Nyberg-Hansen \& Rinvik), entre 65-80 m/s (Ahmed \& Wieraszko, 2012). En los primates, los axones de la vía corticoespinal lateral establecen conexiones con las motoneuronas alfa de las astas anteriores a través de sinapsis monosinápticas directas, mientras que en otras especies de mamíferos no primates la conexión es de tipo multisináptica (Eyre et al.). Esta conexión monosináptica, de relación 1:1, podría ser la base neuroanatómica para explicar por qué aquellos animales que poseen tracto corticoespinal lateral desarrollan un control neurofisiológico del movimiento mucho más fino y preciso. De las fibras que se decusan formando el tracto corticoespinal lateral, la mayoría de ellas hacen sinapsis en la región posterolateral de las astas anteriores (Nyberg-Hansen \& Rinvik). Tales fibras controlan las motoneuronas inferiores, asociadas a la motricidad voluntaria de los miembros y, en el ser humano, están relacionadas específicamente con los movimientos finos de las manos (Nyberg-Hansen \& Rinvik). Los terminales nerviosos de los tractos corticoespinales utilizan el glutamato y el aspartato como neurotransmisores (Penzes \& Remmers, 2012) en las sinapsis neuro-neuronales que se establecen en la médula espinal. Dichos neurotransmisores interactúan con receptores NMDA y AMPA, dependientes de la expresión de Kalirin-7 (Ferraro et al., 2012), ubicados en las motoneuronas alfa espinales. Una vez activados los receptores, se generan potenciales de acción en sus conos axonales, los cuales alcanzan la sinapsis neuromuscular donde se libera finalmente la acetilcolina. En el sarcolema de las fibras musculares se encuentran abundantes receptores colinérgicos (Johnston et al., 2018), que actúan como receptores ionotrópicos, por donde los iones de $\mathrm{Na}+$ fluyen al interior de la fibra muscular provocando su despolarización. A partir de este momento se da inicio a una cascada de reacciones intracelulares, desencadenada por un aumento en la concentración del $\mathrm{Ca} 2+$ citoplasmático, lo cual da como resultado final la contracción muscular (Weavil et al.), que consiste en el deslizamiento de los microfilamentos de miosina sobre los de actina. Esta contractilidad muscular puede ser modificada promoviendo la excitabilidad corticoespinal, mediante la facilitación motoneuronal durante los ciclos no fatigantes, efecto que se elimina durante la fatiga (Bennett \& Hacker).

\section{CONCLUSIÓN}

El interés por conocer la naturaleza decusada de la vía nerviosa para la motricidad voluntaria comenzó con Hipócrates, en la Grecia antigua. A partir de entonces, muchos investigadores, en distintas épocas y latitudes, han aportado valiosos hallazgos al conocimiento neuroanatómico y neurofisiológico que actualmente se tiene de esta vía. El resultado de este conocimiento se ve reflejado en su aplicación en el campo terapéutico, lo que ha permitido mejorar la calidad de vida de pacientes con problemas en su función motora. 
DUQUE, P. J. E.; MENDOZA, Z. J. \& BARCO, R. J. The corticospinal tract: historical perspective. Int. J. Morphol., 38(6):1614-1617, 2020.

SUMMARY: Neuroanatomy and Neurophysiology have, in large part, permitted a more thorough understanding of those structures that conform the nervous system and mechanisms associated with the transmission of action potentials associated with the corticospinal tract. This assertion is made based upon a literature review of various databases and textbooks dedicated to said nerve tracts. The information obtained was ordered chronologically, and data was selected that, from the neuroanatomical and neurophysiological viewpoints, were most relevant and have permitted the comprehension of its functional mechanism. The thorough understanding of those elements that compose the corticospinal tract will permit its application in the health field and resolve multiple motor function problems.

KEY WORDS: History; Neuroanatomy; Corticospinal tract; Neurophysiology; Motor neuron.

\section{REFERENCIAS BIBLIOGRÁFICAS}

Afifi, A. K. \& Bergman, R. A. Neuroanatomía Funcional. Texto y Atlas. $2^{\mathrm{a}}$ ed. Ciudad de México, Mc Graw Hill, 2006.

Ahmed, Z \& Wieraszko, A. Trans-spinal direct current enhances corticospinal output and stimulation-evoked release of glutamate analog, D-2,3-ПНaspartic acid. J. Appl. Physiol. (1985), 112(9):1576-92, 2012.

Al Masri, O. An essay on the human corticospinal tract: history, development, anatomy, and connections. Neuroanatomy, 10:1-4, 2011.

Arráez-Aybar, L. A.; Navia-Álvarez, P.; Fuentes-Redondo, T. \& Bueno-López, J. L. Thomas Willis, a pioneer in translational research in anatomy (on the 350th anniversary of Cerebri anatome ). J. Anat., 226(3):289-300, 2015.

Bennett, M. R. \& Hacker, P. M. S. History of Cognitive Neuroscience. London, Wiley-Blackwell, 2008.

Bravo-Ambrosio, A.; Mastick, G. \& Kaprielian, Z. Motor axon exit from the mammalian spinal cord is controlled by the homeodomain protein Nkx2.9 via Robo-Slit signaling. Development, 139(8):1435-46, 2012.

Davidoff, R. A. The pyramidal tract. Neurology, 40(2):332-9, 1990.

Duque Parra, J. E.; Barco Ríos, J. \& Morales Parra, G. In vivo dissection (vivisection): A historical viewpoint. Int. J. Morphol., 32(1):101-5, 2014.

Duque-Parra, J. E. Elementos neuroanatómicos y neurológicos asociados con el cerebro a través del tiempo. Rev. Neurol., 34(3):282-6, 2002.

Eyre, J. A.; Taylor, J. P.; Villagra, F.; Smith, M. \& Miller, S. Evidence of activity-dependent withdrawal of corticospinal projections during human development. Neurology, 57(9):1543-54, 2001.

Ferraro, E.; Molinari, F. \& Berghella, L. Molecular control of neuromuscular junction development. J. Cachexia Sarcopenia Muscle, 3(1):13-23, 2012.

Finger, S. Origins of Neuroscience. A History of Explorations Into Brain Function. Oxford, Oxford University Press, 1994.

Gross, C. G. The discovery of motor cortex and its background. J. Hist. Neurosci., 16(3):320-31, 2007.

Holodny, A. I.; Watts, R.; Korneinko, V. N.; Pronin, I. N.; Zhukovskiy, M. E.; Gor, D. M. \& Ulung, A. Diffusion tensor tractography of the motor white matter tracts in man: Current controversies and future directions. Ann. N. Y. Acad. Sci., 1064:88-97, 2005.

Hooks, B. M.; Papale, A. E.; Paletzki, R. F.; Feroze, M. W.; Eastwood, B. S.; Couey, J. J.; Winnubst, J.; Chandrashekar, J. \& Gerfen, C. R. Topographic precision in sensory and motor corticostriatal projections varies across cell type and cortical area. Nat. Commun., 9(1):3549, 2018.
Jacobs, B.; Garcia, M. E.; Shea-Shumsky, N. B.; Tennison, M. E.; Schall, M.; Saviano, M. S.; Tummino, T. A.; Bull, A. J.; Driscoll, L. L.; Raghanti, M. A.; et al. Comparative morphology of gigantopyramidal neurons in primary motor cortex across mammals. J. Comp. Neurol., 526(3):496536, 2018.

Johnston, J. R.; Chase, P. B. \& Pinto, J. R. Troponin through the lookingglass: emerging roles beyond regulation of striated muscle contraction. Oncotarget, 9(1):1461-82, 2018.

Kiernan, J. A. \& Rajakumar, N. El Sistema Nervioso Humano. Una Perspectiva Anatómica. $10^{\mathrm{a}}$ ed. Barcelona, Wolters Kluwer/Lippincott Williams \& Wilkins, 2014.

Kraskov, A.; Soteropoulos, D.; Glover, I.; Lemon, R. N. \& Baker, S. N. Slowlyconducting pyramidal tract neurons in macaque and rat. Cereb. Cortex, 2019. DOI: https://www.doi.org/10.1101/768770

Lazar, J. W. Diffusion of electrical current in the experiments of Fritsch and Hitzig and Ferrier failed to negate their conclusion of the existence of cerebral motor centers. J. Hist. Neurosci., 18(4):366-76, 2009.

Lemon, R. N. \& Griffiths, J. Comparing the function of the corticospinal system in different species: organizational differences for motor specialization? Muscle Nerve, 32(3):261-79, 2005.

Markram, H.; Wang, Y. \& Tsodyks, M. Differential signaling via the same axon of neocortical pyramidal neurons. Proc. Natl. Acad. Sci. U. S. A., 95(9):5323-8, 1998

Nathan, P. W.; Smith, M. C. \& Deacon, P. The corticospinal tracts in man. Course and location of fibres at different segmental levels. Brain, 113(Pt. 2):303-24,1990.

Nyberg-Hansen, R. \& Rinvik, E. Some comments on the pyramidal tract, with special reference to its individual variations in man. Acta Neurol. Scand., 38(1):1-30, 1963.

Penzes, P. \& Remmers, C. Kalirin signaling: implications for synaptic pathology. Mol. Neurobiol., 45(1):109-18, 2012.

Rivara, C. B.; Sherwood, C. C.; Bouras, C. \& Hof, P. R. Stereologic characterization and spatial distribution patterns of Betz cells in the human primary motor cortex. Anat. Rec. A Discov. Mol. Cell. Evol. Biol., 270(2):137-51, 2003.

Rothwell, J. C.; Thompson, P. D.; Day, B. L.; Dick, J. P.; Kachi, T.; Cowan, J. M. \& Mandersen, C. D. Motor cortex stimulation in intact man. 1. General characteristics of EMG responses in different muscles. Brain, 110(Pt. 5):1173-90, 1987.

Schieber, M. H. Chapter 2 Comparative anatomy and physiology of the corticospinal system. Handb. Clin. Neurol., 82:15-37, 2007.

Szmuda, T.; Kieron'ska, S.; Ali S.; S?oniewski, P.; Pacholski, M.; Dzierz anowski, J.; Sabisz, A.\& Szurowska, E. Tractography-guided surgery of brain tumors: What is the best method to outline the corticospinal tract? Folia Morphol. (Warsz.), 2020. DOI: https:// www.doi.org/10.5603/FM.a2020.0016

Weavil, J. C.; Sidhu, S. K.; Mangum, T. S.; Richardson, R. S. \& Amann, M. Fatigue diminishes motoneuronal excitability during cycling exercise. $J$. Neurophysiol., 116(4):1743-51, 2016.

Zinger, N.; Harel, R.; Gabler, S.; Israel, Z. \& Prut, Y. Functional organization of information flow in the corticospinal pathway. J. Neurosci., 33(3):11907, 2013.

\section{Dirección para correspondencia: Jorge Eduardo Duque Parra Departamento de Ciencias Básicas Programa de Medicina Universidad de Caldas Manizales COLOMBIA}

\section{Email: jduqueparra@yahoo.com.mx}

Recibido: 28-04-2020

Aceptado: 11-07-2020 